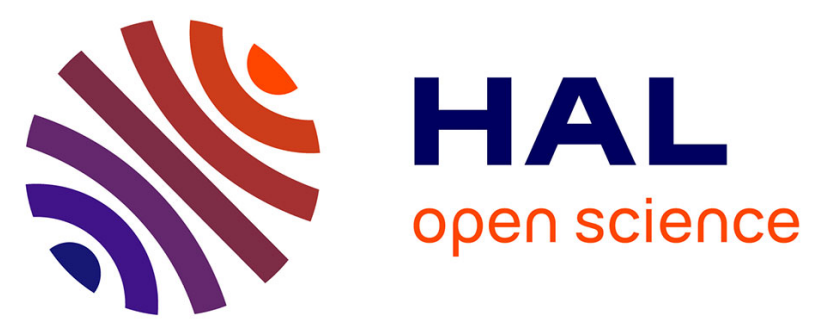

\title{
EFFECT OF SALINITY ON THE DIELECTRIC PROPERTIES OF GEOLOGICAL MATERIALS : IMPLICATION FOR SOIL MOISTURE DETECTION BY MEANS OF REMOTE SENSING
}

Y. Lasne, Philippe Paillou, Gilles Ruffié, Carlos Serradilla, François Demontoux, Anthony Freeman, Tom Farr, K. Mcdonald, B. Chapman, J.-M. Malézieux

\section{- To cite this version:}

Y. Lasne, Philippe Paillou, Gilles Ruffié, Carlos Serradilla, François Demontoux, et al.. EFFECT OF SALINITY ON THE DIELECTRIC PROPERTIES OF GEOLOGICAL MATERIALS : IMPLICATION FOR SOIL MOISTURE DETECTION BY MEANS OF REMOTE SENSING. IEEE Transactions on Geoscience and Remote Sensing, 2008, 46 (6), pp.1674-1688. hal-00155310

\author{
HAL Id: hal-00155310 \\ https://hal.science/hal-00155310
}

Submitted on 18 Jun 2007

HAL is a multi-disciplinary open access archive for the deposit and dissemination of scientific research documents, whether they are published or not. The documents may come from teaching and research institutions in France or abroad, or from public or private research centers.
L'archive ouverte pluridisciplinaire HAL, est destinée au dépôt et à la diffusion de documents scientifiques de niveau recherche, publiés ou non, émanant des établissements d'enseignement et de recherche français ou étrangers, des laboratoires publics ou privés. 


\section{EFFECT OF SALINITY ON THE DIELECTRIC PROPERTIES OF GEOLOGICAL MATERIALS : IMPLICATION FOR SOIL MOISTURE DETECTION BY MEANS OF REMOTE SENSING}

\author{
Y. Lasne, Ph. Paillou, G. Ruffié, C. Serradilla, F. \\ Demontoux \\ IMS Laboratory, ENSCPB \\ 33607 Pessac, FRANCE \\ yannick.lasne@ims-bordeaux.fr
}

\author{
A. Freeman, T. Farr, K. McDonald, B. Chapman \\ Jet Propulsion Laboratory, NASA \\ Pasadena, CA 91109, USA \\ J.-M. Malézieux \\ EGID Institute \\ 33607 Pessac, FRANCE
}

\begin{abstract}
This paper deals with the exploitation of dielectric properties of saline deposits for the detection and mapping of moisture in arid regions on both Earth and Mars. We then present a simulation and experimental study in order to assess the effect of salinity on the permittivity of geological materials and therefore on the radar backscattering coefficient in the $[1-7 \mathrm{GHz}]$ frequency range. Dielectric mixing models were first calibrated by means of experimental measurements before being used as input parameters of analytical scattering models (IEM, SPM). Simulation results will finally be compared to field measurements (Pyla dune, Death Valley, Mojave Desert) and will be used for the interpretation of SAR data (AIRSAR, PALSAR).
\end{abstract}

Keywords- Earth, evaporites, dielectric mixing model, IEM, Mars, polarimetry, radar backscattering, salinity, SAR, soil moisture.

\section{INTRODUCTION}

The measurement of soil moisture by means of radar systems has been widely investigated over the last two decades [1-6]. As the electric properties of soils depend on their water content, it impacts the radar backscattering coefficient $\boldsymbol{\sigma}^{\mathbf{0}}$ [7-8]. While the effect of water content on the permittivity has been widely studied and modeled, little has been done on the salinity effect. Recent work has underlined the influence of the presence of salts in solution on the dielectric properties of soils and especially on the imaginary part $\boldsymbol{\varepsilon}$ " of the dielectric constant [9-11]. If the real part $\boldsymbol{\varepsilon}$ ' of the permittivity, related to the medium polarization, governs the velocity of propagation of a wave through the material, $\boldsymbol{\varepsilon}$ " is related to the conductivity of the medium and represents the radar signal attenuation by energy absorption required to reach the polarization (ohmic losses). It thus appears essential to take into account the occurrence of saline deposits (evaporites) in radar remote sensing applications. We have started to study the influence of salts on the backscattering coefficient $\boldsymbol{\sigma}^{\mathbf{0}}$ through the dielectric properties of the material. The adopted methodology is based on measurements carried out in the laboratory by means of a vectorial network analyzer in order to firstly assess the validity of dielectric mixing models [12-15], based on the Debye formulas. These dielectric models will then provide input parameters required for the modeling of $\boldsymbol{\sigma}^{\mathbf{0}}$ by means of analytical scattering models (IEM, SPM) [5-6], [16-19]. Simulation results will finally be compared to field measurements (Pyla dune, Death Valley, Mojave Desert) and will be used for the interpretation of SAR data (AIRSAR, PALSAR). Our objective is to exploit the dielectric properties of saline deposits for the detection and mapping of moisture in arid regions on both Earth and Mars (nowadays the occurrence of evaporites on Mars is commonly accepted [20]).

\section{EVAPORITES}

Evaporites are sedimentary deposits resulting from a chemical precipitation of dissolved substances in natural solutions (continental or marine) with an increase in their concentrations by terrigenous contributions and evaporation [21]. The most important concentration factor lies in evaporation processes although congelation and hydrothermal processes can also lead to supersaturated conditions necessary to mineral precipitation. The formation and the paragenesis of saline deposits are thus closely related to the physicochemical properties of minerals in solution (in terms of solubility) and to the parameters of evaporitic environments (temperature, salinity, evaporation rate, hydrostatic differential, $\mathrm{pH}$, microbial activity).

\section{DIELECTRIC MIXING MODELS}

The influence of saline deposits in radar remote sensing is related to the solubility behavior and the ionic properties of the minerals. In their ionic (dissolved) form, the weight of dissolved substances per kilogram of solution defines the conductivity of the solution. This conductivity is directly related to the presence of free electrons and ions [22]. Under the action of an electric field, the distribution of charges is distorted and the barycentres of positive and negative charges no longer coincide. This results in an induced dipole moment that can be interpreted as the polarization vector $\boldsymbol{P}$. In this case, we will be interested in the microscopic interpretation of polarization: atoms, molecules, ions. The presence of such components generates three microscopic polarization mechanisms: electronic, ionic, and orientation polarizabilities, which can be related to the medium permittivity (Clausius-Mossotti relation for polar molecules). More precisely, $\boldsymbol{\varepsilon}$ " being proportional to the conductivity (interaction of free charges), we have to evaluate the impact of the presence of salts on the dielectric properties of the medium.

Assuming a multiphase approach for the mixtures (soil particle, free water, bound water, air voids, salts), we initially considered the dielectric mixing models proposed by Dobson [12] and Wang [15] to assess the influence of salinity on the dielectric properties of geological materials. Firstly, these models have been applied to the case of a sand/salt/water mixture corresponding to a test site we are working on: the Pyla dune [1, 23-24]. As sea water contains mainly $\mathrm{NaCl}$, we considered results obtained for $\mathrm{NaCl}$ solutions as a first approximation allowing the application of the Stogryn equations [5, 13] for the electromagnetic characterization of sea water [25].

Contrarily to the Dobson model, the semi-empirical model proposed by Wang at 1.4 and $5 \mathrm{GHz}$ allows to distinguish two regions for the variations of the dielectric constant as a function of the mixture moisture. When $m_{v}<W_{t}, W_{t}$ being the transition 
moisture, water molecules are tightly bound to the soil particles. Making comparison to ice molecules, Wang has shown similarity in activation energy values. Consequently, we have considered the dielectric properties of ice as a first approximation for those of bound water. By opposition, for moisture values higher than the transition moisture $\left(m_{v}>W_{t}\right)$, water can be considered as free and its dielectric constant can be described by means of the classical equations of the Debye theory for a polar medium. Assuming a 4component dielectric mixing model, expressions of the complex permittivity are given by:

$$
\varepsilon=m_{v}+\left(P-m_{v}\right) \varepsilon_{a}+(1-P) \varepsilon_{r}
$$

for $m_{v}<W_{t}$, with:

$$
\varepsilon_{x}=\varepsilon_{i}+\left(\varepsilon_{w}-\varepsilon_{i}\right) \cdot \frac{m_{v}}{W_{t}} \cdot \gamma
$$

and

$$
\varepsilon=W_{t} \varepsilon_{x}+\left(m_{v}-W_{t}\right) \varepsilon_{w}+\left(P-m_{v}\right) \varepsilon_{a}+(1-P) \varepsilon_{r}
$$

for $m_{v}>W_{t}$, with

$$
\varepsilon_{x}=\varepsilon_{i}+\left(\varepsilon_{w}-\varepsilon_{i}\right) \gamma
$$

where $P$ denotes the porosity. $\varepsilon_{a}, \varepsilon_{w}, \varepsilon_{r}$, and $\varepsilon_{i}$ are the dielectric constants of air, water, soil particles and ice respectively. $\varepsilon_{x}$ corresponds to the complex permittivity of bound water. $W_{t}$ and $\gamma$ being soil texture dependent (volume fraction of sand, clay, loam...), they have been assessed on Pyla dune samples.

In order to take into account losses due to the ionic conductivity of samples (because of the presence of ions in the free water component), the dielectric properties of the $\mathrm{NaCl}$ solution have been determined using the Debye's classical theory modified by Lane and Saxton [26]. According to the Stogryn formulation, real and imaginary parts of the saline solution are given by $[5,13]$ :

$$
\begin{gathered}
\varepsilon_{s w}^{\prime}=\varepsilon_{s w \infty}+\frac{\varepsilon_{s w o}-\varepsilon_{s w \infty}}{1-\left(2 \pi \tau_{s w} f\right)^{2}} \\
\varepsilon_{s w}^{\prime \prime}=\left(2 \pi \tau_{s w} f\right) \cdot \frac{\varepsilon_{s w o}-\varepsilon_{s w \infty}}{1-\left(2 \pi \tau_{s w} f\right)^{2}}+\frac{\sigma_{i}}{2 \pi f \varepsilon_{o}}
\end{gathered}
$$

where $\tau_{s w}$ and $\sigma_{i}$ correspond to the relaxation time and the ionic conductivity of the $\mathrm{NaCl}$ solution and are temperature as well as salinity dependent [13].

The permittivity of the sand/sea water mixture was computed according to the frequency (L- and C-Bands), the moisture content $\left(m_{v}<0.6\right)$, and the salinity $(\mathrm{S}<140 \%$ oo). The temperature was fixed at $20^{\circ} \mathrm{C}$, the bulk density is assumed to be $1.74 \mathrm{g.cm}$, and the specific density of the solid fraction $2.65 \mathrm{~g} . \mathrm{cm}^{-3}$ (Figs. 1, 2). The other constants correspond to those found in the literature $[5,13]$.

\section{A. Salinity Effect on the Dielectric Constant}

Our first results show that if the real and imaginary parts increase with the moisture content, the impact of salinity isn't the same for $\boldsymbol{\varepsilon}^{\prime}$ and $\boldsymbol{\varepsilon}$ '. As the real part is concerned, the results presented in Fig. 1 shows that soil salinity has a little influence on $\boldsymbol{\varepsilon}^{\prime}$ except for soil with high moisture content $\left(m_{v}>0.2\right)$ for which $\boldsymbol{\varepsilon}^{\prime}$ decreases with increasing salinity. For small salinity values, the increasing moisture content appears to be the main factor in $\boldsymbol{\varepsilon}^{\prime}$ variations. On the contrary, the imaginary part $\boldsymbol{\varepsilon}^{\prime \prime}$ is strongly affected by both salinity and moisture of soil samples. In particular, the higher the magnitude of humidity the stronger the effect of salinity on the imaginary part. Since $\varepsilon^{\prime \prime}$ is proportional to the

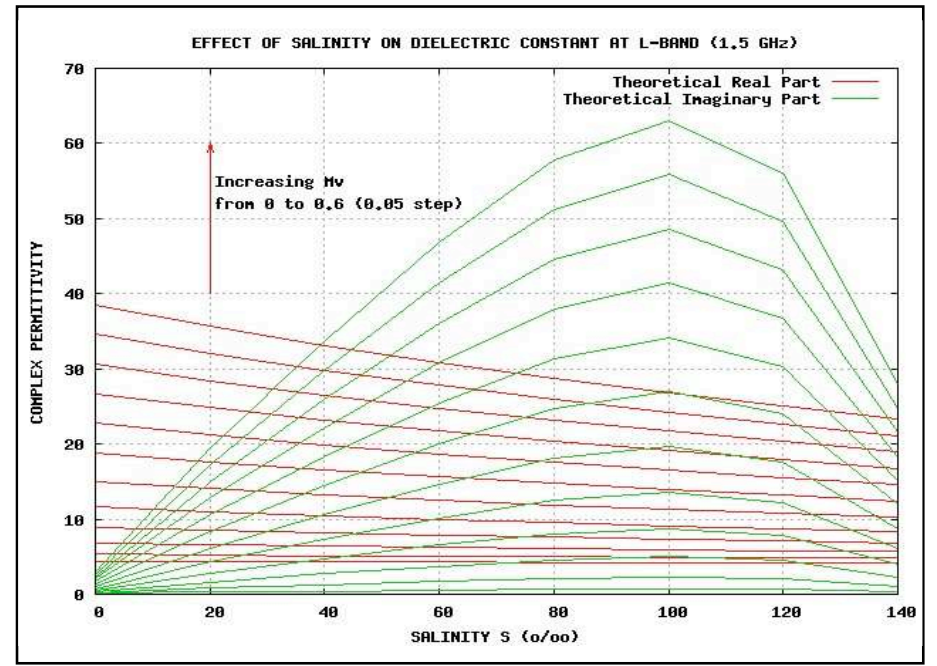

Fig. 1: Effect of salinity on the complex permittivity of a sand/saline water mixture derived from the Wang's model at $1.5 \mathrm{GHz}$.

conductivity, such a behavior can be explained by the increase in soil moisture content that leads to a greater amount of salts dissolved in the soil system (greater free water component) and thus to the increase in conductivity. More precisely, for small water content, the bound water component dominates leading to a weak increase in dielectric constant since its dielectric properties are closed to the ice ones. For increasing water content values, the free water component becomes much more effective allowing a greater amount of dissolved salts and then a higher conductivity. Fig. 1 also clearly indicates that the rate of increase in $\varepsilon$ " with the salinity is dependent on soil moisture content. Nevertheless, when salt concentrations are in excess $\left(S>100 \%\right.$ oo), $\boldsymbol{\varepsilon}^{\prime \prime}$ decreases. This can be related to a precipitation of dissolved salts leading to a decrease in free water conductivity. This behavior confirms the one reported in recent works of Mironov [14] on bentonite, Shao [10] on $\mathrm{NaCl}$ solutions, and Aly [9] on silty loam soil samples.

Assuming a transition moisture content, the semiempirical model proposed by Wang seems to provide a better description of the dielectric constant measurements. For such a reason, we preferred in the following the semiempirical approach of Wang to the Dobson one in order to characterize the dielectric properties of the sand/ saline water mixture

\section{B. Frequency Effect on the Dielectric Constant}

Since the conductivity depends on the frequency, we aim at assessing the frequency effect on the dielectric constant of our moistened and salinized mixture by means of the Wang semiempirical model.

If the results for the real part indicate that $\varepsilon^{\prime}$ slowly decreases with increment of frequency for high content moisture $\left(m_{v}>20 \%\right)$ whatever the salinity value may be, the most interesting results concern the imaginary part of the soil mixture related to the ionic conductivity of the $\mathrm{NaCl}$ electrolyte solution. Actually, it can be seen on Fig. 2 that $\boldsymbol{\varepsilon}^{\prime \prime}$ steeply decreases in the [1-4 GHz] frequency range in the presence of salinity $(S>0)$ while $\varepsilon$ " increases when $S$ $=0$. Moreover, Shao [10] reported the same $\varepsilon^{\prime \prime}$ variations derived from experimental measurements performed on $\mathrm{NaCl}$ concentrated solutions. Likewise, investigations carried out by Komarov [27] led to the same conclusions concerning kaolinite, montmorillonite and $\mathrm{NaCl}$ that may be attributed to the influence of the relaxation time of the saturated solution [12, 26, 28, 29]. According to the DebyeFalkenhagen theory, the variations of $\varepsilon^{\prime \prime}$ with respect to the frequency could be attributed to the frequency dependence of the ionic conductivity which usually describes the motion of ions in an 

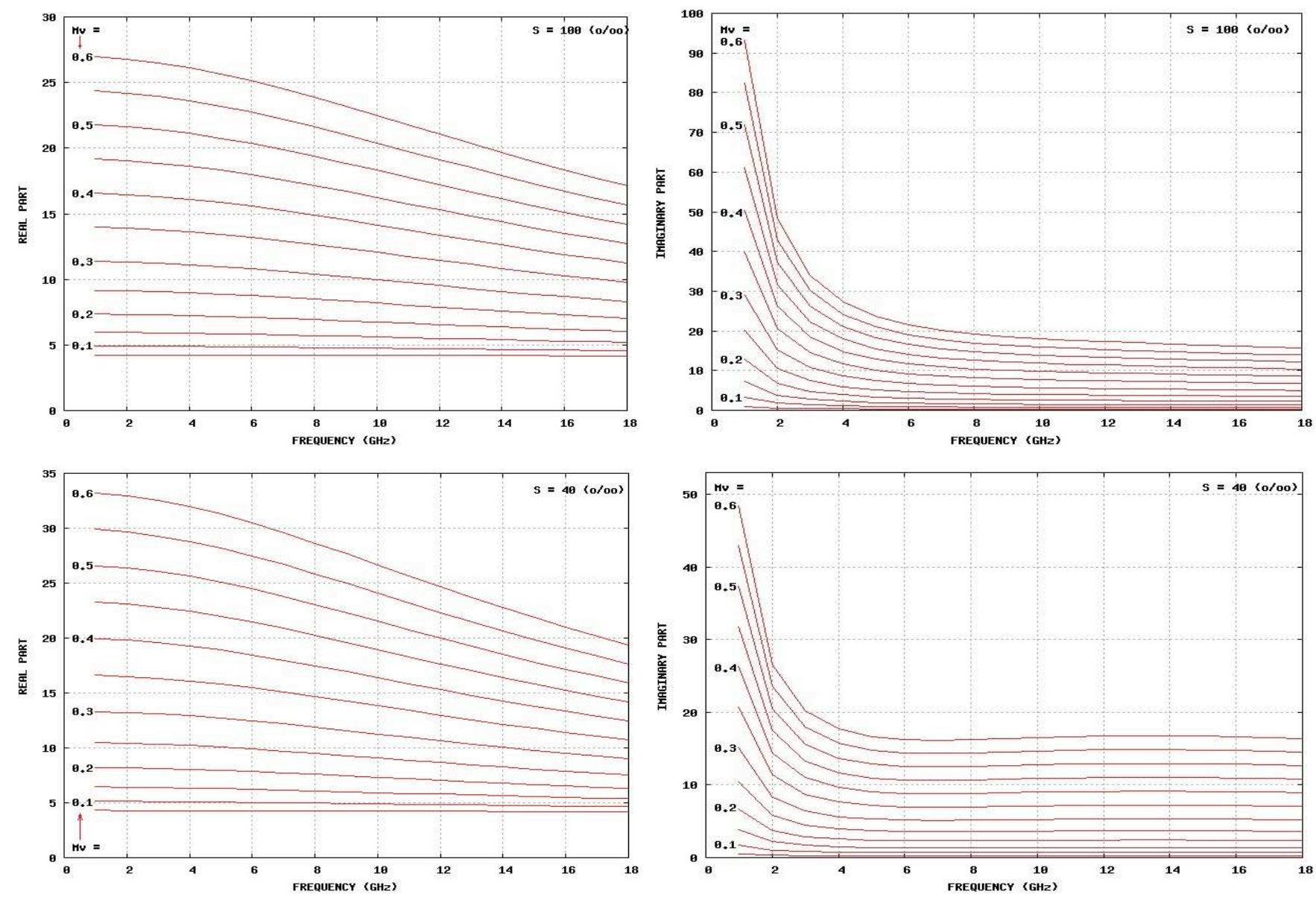

Fig. 2: Frequency variations of real (left) and imaginary (right) parts of the dielectric constant of the sand/saline water mixture derived from the Wang's model for several moisture content and fixed salinity values $S=100 \%$ (at the top) and $S=40 \%$ (at the bottom).

electrolyte solution [28]. In particular, Chandra [29] presented a theory for the frequency dependence of the ionic conductivity of an electrolyte solution where the ionic conductivity decreases at high frequency. These authors considered the dynamic effect of the relaxation of an ion atmosphere (space of opposite sign of charge which surrounds the central ion) on the motion of an ion. Moving in an electrolyte solution, an ion is subject to a retarding force due to the ionic atmosphere lags. When an ion moves, through the liquid with a velocity produced by an external electric field, the ionic atmosphere cannot immediately follow the motion leading to a dissymmetry in the direction of the ion motion. This dissymmetry of the electric density causes a force (electric force of relaxation [28]) on the moving ion which decreases its mobility. Assuming an oscillating electric field (microwave emission), the central ion oscillates and the ion atmosphere gets less time to reach the relaxation (relaxation time). The atmosphere is then less asymmetric. As a result, the effect of the electric force of relaxation is reduced leading to a higher ion mobility (reduction of electrolyte friction) and hence an enhancement of the conductivity at low frequency. At higher frequency, the dissymmetry of the ionic atmosphere cannot be built up since the central ion oscillates so fast that the net ionic motion is smaller than the one in the presence of a static or low frequency electric field. As a result, the retarding effect vanishes and the ionic atmosphere as well as the central ion can be regarded as motionless leading to a decrease in ion mobility and then in the ionic conductivity [28, 29]. Moreover, it was shown that at high frequency, the dominant loss mechanism on the dielectric constant is mainly due to the dielectric relaxation (polarization losses) of free water since the maximum of $\boldsymbol{\varepsilon}^{\text {" occurs }}$ at the relaxation frequency $[27,30](17 \mathrm{GHz}$ for pure water): it could explain the increase in $\boldsymbol{\varepsilon}^{\prime \prime}$ observed by Hallikainen [31]. On the contrary, at lower frequency [1-4 GHz], the ionic conductivity is the main contributor in dielectric losses causing $\varepsilon$ " of moistened salinized soils to vary inversely with frequency. Between these two regimes, $\boldsymbol{\varepsilon}^{\prime \prime}$ would be expected to pass through a minimum [30] especially for small salinity and high moisture content values as reported by [10] and [12].

\section{LABORATORY MEASUREMENTS}

In order to determine the validity of the previous dielectric mixing model, we performed complex permittivity measurements for a sand/sea water mixture with the same frequency range, moisture content and salinity values.

Measurements were carried out by means of a vectorial network analyzer coupled to an open-ended coaxial probe (SMA type) providing the reflexion coefficient S11 (amplitude and phase) of the electromagnetic wave at the sample surface [32].

Since the electromagnetic field at the probe/sample interface can be represented by a capacitive equivalent circuit (capacitor), a calibration procedure performed on standard chemical solutions (for which the variations of the complex permittivity with the frequency are well known) allows to define the probe parameters. The knowledge of such parameters combined with measurements of the S11 reflection coefficient then makes it possible to retrieve real and imaginary parts of the dielectric constant of geological material samples using a capacitive inversion algorithm.

Fig. 3 displays the measurements results of real and imaginary parts of sand/saline water mixture as function of frequency and moisture for two salinity values $(S=40$ and $100 \%$ o). These results 


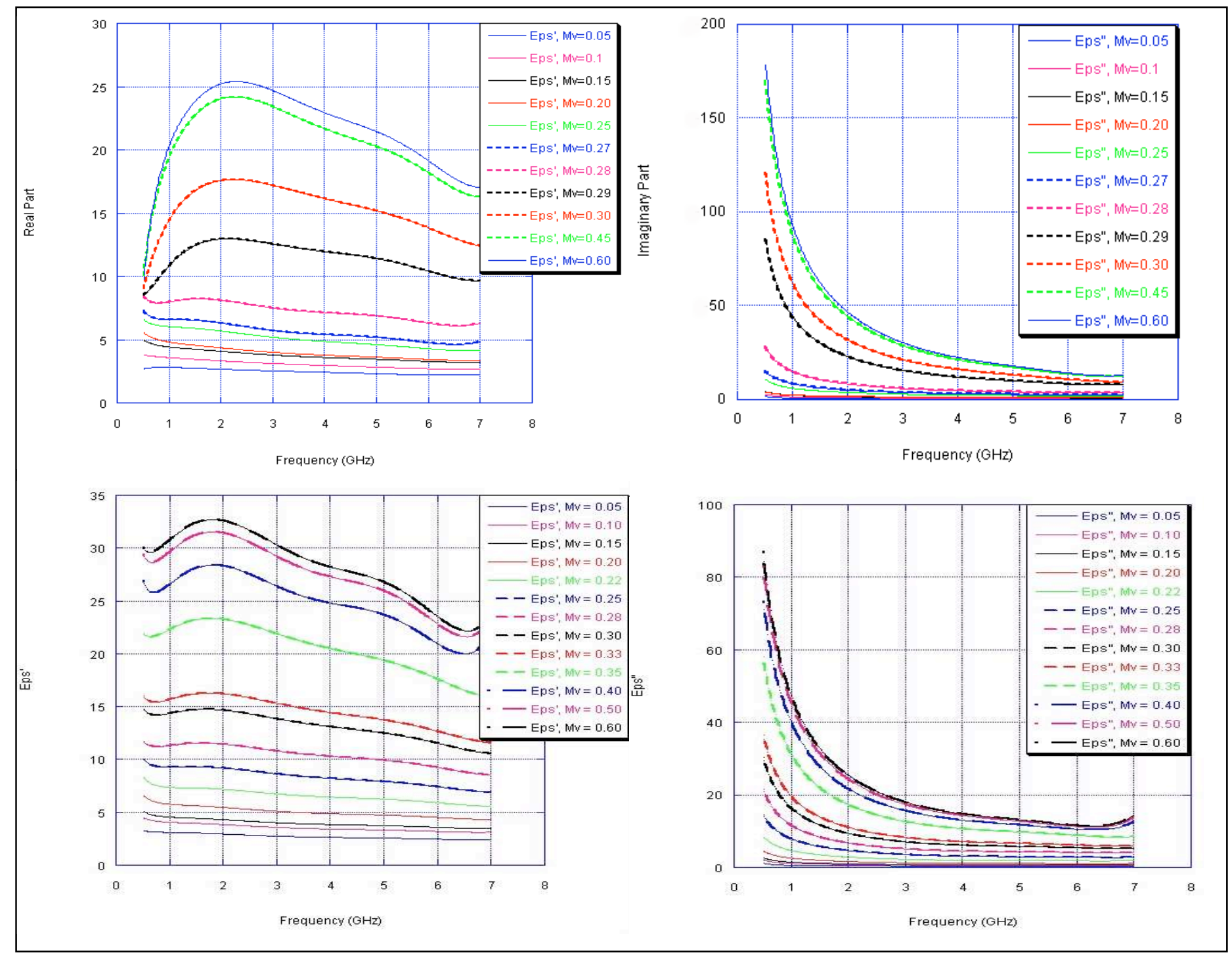

Fig. 3: Frequency variations of real (left) and imaginary (right) parts of the dielectric constant of the sand/saline water mixture derived from laboratory measurements for several moisture content and fixed salinity values $S=100 \%$ (at the top) and $\mathrm{S}=40 \%$ (at the bottom)

confirm the predicted salinity and frequency dependence of the dielectric constant. As the real part is concerned, measurements results clearly indicate that $\boldsymbol{\varepsilon}^{\prime}$ decreases with increment in salinity as well as in frequency. Moreover, $\boldsymbol{\varepsilon}^{\prime}$ values derived from the Wang model are in good agreement with those derived from measurements in the $[1-3 \mathrm{GHz}]$ frequency range. Dealing with the imaginary part, laboratory results clearly indicate the influence of salinity on $\varepsilon^{\prime \prime}$ leading to a strong increase of the imaginary part with increment of salinity. Laboratory measurements also confirm the theoretical frequency dependence of $\varepsilon "$ displaying a steep variation rate in the $[1-2 \mathrm{GHz}]$ frequency range although the theoretical values appear to underestimate the measured ones. It would thus be necessary to make some modifications to the dielectric mixing model according to experimental data sets.

Nevertheless, it should be noticed that the experimental results showing a transition moisture value $\left(W_{t} \sim 29 \%\right.$ for $S=100 \%$ and $\sim 33 \%$ for $S=40 \%$ o), they confirm the bimodal approach proposed by Wang [15] which will then be used on the following.

\section{NUMERICAL SIMULATIONS OF THE BACKSCATTERING COEFFICIENT}

In order to assess the impact of salts on airborne or orbital SAR data, we computed the radar backscattering coefficients at L-Band $(1.5 \mathrm{GHz})$ for the complex permittivity values previously measured. Assuming smooth surfaces (rms-height of $0.3 \mathrm{~cm}$ and correlation length of $2.7 \mathrm{~cm}$ ), we used the analytical IEM and SPM scattering models. Considering the surface scattering term only, the single backscattering coefficient is expressed by:

$$
\sigma_{\text {SSpp }}^{0}(\theta)=\frac{k}{4} e^{-2 k^{2} \cos ^{2}(\theta) \sigma^{2}} \sum_{n=1}^{\infty}\left|I_{p p}^{n}\right|^{2} \cdot \frac{W^{n}(2 k \sin (\theta))}{n !}
$$

for the IEM scattering model, and :

$$
\sigma_{p p}^{0}=8 k^{4} \sigma^{2} \cos ^{4}(\theta) \cdot\left|\alpha_{p p}\right|^{2} \cdot W(2 k \sin (\theta), 0)
$$

for the SPM. $p p$ indicates the polarization state of the emitted/scattered wave respectively ( $\mathrm{HH}$ or VV), $\theta$ is the incident angle, $k$ the wavenumber, $W^{n}$ the Fourier transform of the $n$th power of the surface correlation function and $\alpha_{\mathrm{pp}}$ are the Fresnel reflexion coefficients.

Results presented in Fig. 4 indicate that the sensitivity of the backscattering coefficient to the salinity depends on the moisture content. This sensitivity is more important for the vertical polarization (VV). Although salinity more slightly affects the horizontal polarization $(\mathrm{HH})$, in particular for high moisture contents, the strong dependence of the backscattering coefficient on the salinity for low moisture values constitutes an important result for the detection of small amounts of water when salt is present. Assuming a typical instrument detection limit of about -30dB for an orbital radar, this result is particularly interesting for the Martian case where the expected moisture variations would not exceed a few percent [33-35]. 


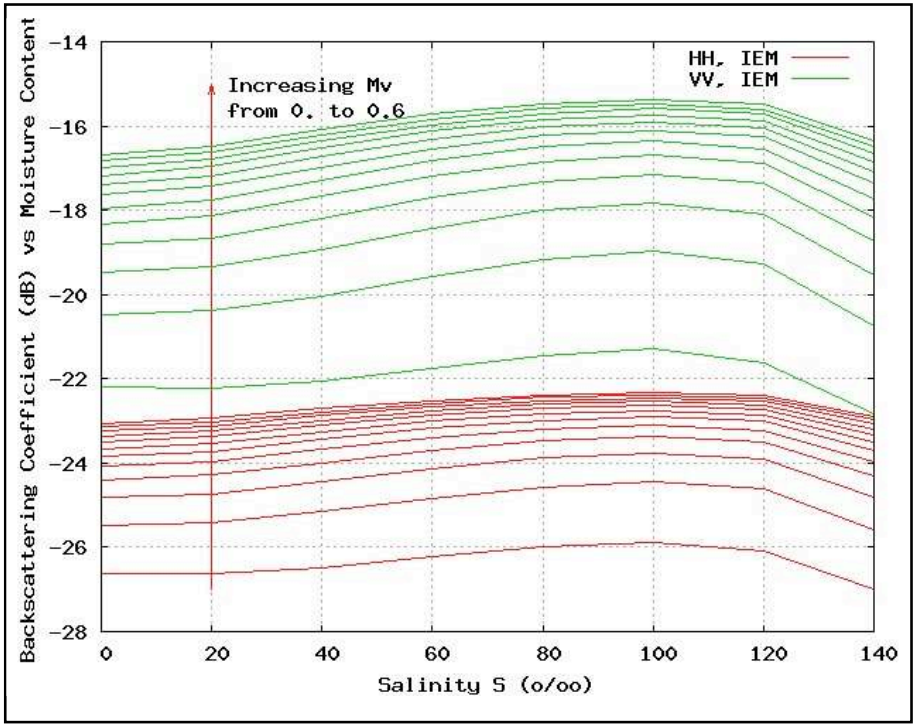

Fig. 4: IEM simulations for the backscattering coefficient at L-Band at $40^{\circ}$ incidence angle.

\section{FUTURE WORK}

We initially considered the influence of salinity on the amplitude of the radar backscattering. As the copolar phase difference is a potential indicator for soil moisture detection [1, 24], we will also study the impact of salinity on radar phase variations.

\section{REFERENCES}

[1] Y. Lasne, Ph. Paillou, Th. August-Bernex, G. Ruffié, and G. Grandjean, "A phase signature for detecting wet subsurface structures using polarimetric L-band SAR," IEEE TGRS, vol. 42, pp. 1683-1694, 2004.

[2] R. Bindlish, and A. P. Barros, "Subpixel variability on remotely sensed soil moisture: An inter-comparison study of SAR and ESTAR," IEEE TGRS, vol. 40, pp. 326-337, 2002.

[3] J. Shi, J. Wang, A. Y. Hsu, P. E. O'Neill, and E. T. Engman, "Estimation of bare surface soil moisture and surface roughness parameter using L-Band SAR image data," IEEE TGRS, vol. 35, pp. 1254-1266, 1997

[4] F. T. Ulaby, P. P. Batlivala, and M. C. Dobson, "Microwave backscatter dependence on surface roughness, soil moisture, and soil texture, Part I Bare soil," IEEE TGE, vol. GE-16, pp. 286-295, 1978.

[5] F. T. Ulaby, R. K. Moore, and A. K. Fung, Microwave Remote Sensing: Active and Passive, Norwood, MA: Artech House, 1981.

[6] A.K. Fung, Microwave Scattering and Emissions Models and Their Applications, Norwood, MA: Artech House, 1994.

[7] P. Dubois, J. van Zyl, and T. Engman, "Measuring soil moisture with imaging radar," IEEE TGRS, vol. 33, pp. 915-926, 1995.

[8] Z. Su, P. A. Troch, and F. P. De Troch, "Estimation of surface soil moisture by inversion of SAR data, surface scattering from random rough, bare soils, spatial and temporal soil moisture mapping from ERS$1 / 2$, and JERS-1 SAR data and macroscale hydrologic modeling for regional climate models (RCM)," ESA, Noordwijk, The Netherlands, ESA Project A02-B106, 1997

[9] Z. Aly, F. Bonn, and R. Magagi, "Modelling the backscattering coefficient of salt-affected soils: applications to Wadi el Natrun bottom, Egypt," EARSel Proceedings 3, pp. 372-381, 2004.

[10] Y. Shao, Q. Hu, H. Guo, Y. Lu, Q. Dong, and C. Han, "Effect of dielectric properties of moist salinized soils on backscattering coefficients extracted from RADARSAT image," IEEE TGRS, vol. 41, pp. 18791888, 2003.

[11] K. Sreenivas, L. Venkataraknam, and P. V. Narasimha Rao, "Dielectric properties of salt-affected soils," Int. J. Remote Sens., vol. 16, pp. 641649, 1994.
[12] M. C. Dobson, F. T. Ulaby, M. T. Hallikainen, and M. A. El-Rayes, "Microwave dielectric behavior of wet soil - Part II: Dielectric mixing models," IEEE TGRS, vol. GE-23, pp. 35-46, 1985.

[13] A. Stogryn, "Equations for calculating the dielectric constant of saline water," IEEE TMTT, pp.733-736, 1971.

[14] V. L. Mironov, M. C. Dobson, V. H. Kaupp, S. A. Komarov, and V. N. Kleshchenko, "Generalized refractive mixing dielectric model for moist soils," IEEE TGRS, vol. 42, pp. 773-785, 2004.

[15] J. R. Wang, and T. J. Schmugge, "An empirical model for the complex dielectric permittivity of soils as a function of water content," IEEE TGRS, vol. GE-18, pp. 288-295, 1980.

[16] A. K. Fung, Z. Li, K. S. Chen, "Backscattering from a randomly rough dielectric surface," IEEE TGRS, vol. 30, pp. 356-369, 1992.

[17] C. Y. Hsieh, A. K. Fung, G. Nesti, A. J. Sieber, and P. Coppo, "A further study of the IEM surface scattering," IEEE TGRS, vol. 35, pp. 901-909, 1997.

[18] K. S. Chen, T. D. Wu, M. K. Tsay, and A. K. Fung, "A note on the multiple scattering in an IEM model," IEEE TGRS, vol. 38, pp. 249-256, 2000.

[19] T. D. Wu, and K. S. Chen, "A reappraisal of the validity of the IEM model for backscattering from rough surfaces," IEEE TGRS, vol. 42, pp. 743-753, 2004

[20] N. J. Tosca, S. M. McLennan, B. C. Clark, J. P. Grotzinger, J. A. Hurowitz, B. L. Jolliff, A. H. Knoll, C. Schröder, S. W. Squyres, "Geochemical modeling of evaporites on Mars: Insight from Meridiani Planum," Workshop LPS XXXVI, 2005.

[21] J. M. Rouchy, and M. M. Blanc-Valleron, Les Evaporites, Vuibert, 208p, 2006.

[22] J. P. Pérez, R. Carles, and R. Fleckinger, Electromagnétisme, Masson, $776 \mathrm{p}, 1997$.

[23] G. Grandjean, Ph. Paillou, P. Dubois, Th. August-Bernex, N. Baghdadi, and J. Achache, "Subsurface structures detection by comining L-band polarimetric SAR and GPR data: Example of the Pyla dune (France)," IEEE TGRS, vol. 39, pp. 1245-1258, 2001.

[24] Y. Lasne, Ph. Paillou, G. Ruffié, and M. Crapeau, "Effect of multiple scattering on the phase signature of wet subsurface structures: Applications to polarimetric L- and C-band SAR," IEEE TGRS, vo. 43, pp. 1716-1726, 2005

[25] J. B. Hasted, D. M. Ritson, and C. H. Collie, "Dielectric properties of aqueous ionic solutions. Parts I and II," J. Chem. Phys. , vol. 16, pp. 1-21, 1948.

[26] J. A. Lane, and J. A. Saxton, "Dielectric dispersion in pure polar liquids at very high radio frequencies. III. The effect of electrolytes in solution," Proc. Roy. Soc., vol. 213, pp. 531-545, 1952.

[27] S. A. Komarov, V. L. Mironov, and A. N. Romanov, "Frequency dispersion in microwave for complex permittivity of bound water stored in soils and wet salts," IGARSS'99, vol. 5, pp. 2643-2645, 1999.

[28] H. Falkenhagen, Reviews of Modern Physics, vol. 3, pp. 412-426, 1931.

[29] A. Chandra, and B. Bagchi, "Frequency dependence of ionic conductivity of electrolyte solutions," Journal of Chemical Physics, vol. 112, pp. 1876-1886, 2000.

[30] J. Sabburg, J. A. R. Ball, and N. H. Hancock, "Dielectric behavior of moist swelling clay soils at microwave frequencies," IEEE TGRS, vol. 35, pp. 784-787, 1997.

[31] M. Hallikainen, F. T. Ulaby, M. C. Dobson, M. A. El-Rayes, and L. K. Wu, "Microwave dielectric behavior of wat soil - Part I: empirical models and experimental observations," IEEE TGRS, vol. GE-23, pp. 25-34, 1985.

[32] E. Bouynet, Ph.D. Thesis, University Bordeaux 1, 132p.

[33] W. C. Feldman, T. H. Prettyman, S. Maurice, J. J. Plaut, and D. L. Bish, "Global distribution of near surface hydrogen on Mars," J. Geophys. Res., vol. 109, pp. 1-13, 2004.

[34] I. G. Mitrofanov, M. L. Litvak, A. S. Kozyref, A. B. Sanin, V. Tretyakov, and W. V. Boynton, "Vertical distribution of shallow water in Mars subsurface from HEND/Odyssey data," Microsymposium 38, MS069, 2003.

[35] D. T. Möhlmann, "Water in the upper Martian surface at mid- and lowlatitude: presence, state, and consequences," Icarus, vol. 168, pp. 318323,2004 